\title{
Predictive attenuation of touch and tactile gating are distinct perceptual phenomena
}

\author{
Konstantina Kilteni $^{{ }^{*}}$ and H. Henrik Ehrsson ${ }^{1}$ \\ ${ }^{1}$ Department of Neuroscience, Karolinska Institutet, Solnavägen 9, 17165 Stockholm, Sweden \\ *Correspondence and requests for materials should be addressed to konstantina.kilteni@ki.se
}

In recent decades, research on somatosensory perception has led to two important observations. First, self-generated touches that are predicted by voluntary movements become attenuated compared to externally generated touches of the same intensity (attenuation). Second, externally generated touches feel weaker and are more difficult to detect during movement than at rest (gating). Researchers today often consider gating and attenuation to be the same suppression process; however, this assumption is unwarranted because, despite more than forty years of research, no study has combined them in a single paradigm. We quantified how people perceive self-generated and externally generated touches during movement and rest. We demonstrate that whereas voluntary movement gates the precision of both self-generated and externally generated touch, the amplitude of self-generated touch is selectively attenuated compared to externally generated touch. We further show that attenuation and gating do not interact and are not correlated, and we conclude that they represent distinct perceptual phenomena.

Let us imagine you are at your doctor for a medical examination. Upon her request, you apply pressure with your index finger to your leg to indicate where exactly you feel the pain. The pressure you feel on your leg and the tip of your finger is the feedback from your voluntary finger movement, and it is called somatosensory reafference. To reproduce and confirm your sensations, imagine that the doctor now applies pressure with her index finger to the same spot on your leg. This pressure is generated by the doctor and not by you, and it is called somatosensory exafference. Now imagine that the doctor asks you to first keep your leg relaxed and then flex and extend it while you or her continuously apply pressure to your leg. You can therefore experience your reafferent or her exafferent touches on your leg while it is moving or resting. Distinguishing between these four conditions is fundamental for your sensorimotor control; your nervous system must know both the source of the touch and the state of your limb to make appropriate use of the sensory feedback. A cutaneous mechanoreceptor in your peripheral nervous system, however, cannot distinguish whether a touch is reafferent or exafferent, and thus, this distinction must be made centrally, where tactile signals from the skin, sensory information from muscles and joints, and information from motor commands are available. How, then, does the central nervous system classify somatosensory signals during movement?

Several experimental studies in humans have shown that the brain attenuates somatosensory reafference compared to exafference. In behavioral research, this refers to participants perceiving self-generated strokes, forces, or taps as weaker than external equivalents of the same intensity ${ }^{1-10}$. This somatosensory attenuation has been related to reduced activity in the secondary somatosensory cortex ${ }^{11-13}$ and the cerebellum ${ }^{11,13,14}$ and increased connectivity between the two areas ${ }^{13,15}$ during self-generated touches compared to externally generated touches. Somatosensory attenuation was found in $98 \%$ or 315 out of 322 people across a wide age range ${ }^{16}$, and it is considered one of the reasons why we cannot tickle ourselves ${ }^{17-19}$. 
Sensory attenuation is not exclusive to humans; similar strategies are used by other species across the animal kingdom (for reviews, see ${ }^{20-24}$ ). For example, during self-chirping, the cricket's central auditory processing is inhibited (both presynaptically and postsynaptically) in phase with the insect's chirps to prevent desensitization of its auditory system while maintaining sensitivity to external sounds ${ }^{25,26}$. In mice, auditory cortical responses in response to self-generated sounds are attenuated, and this attenuation is present only for the tone frequencies the animal has associated with its locomotion and absent when the same sounds are externally produced ${ }^{27}$. A weakly electric fish ${ }^{28}$ is able to respond exclusively to externally generated electrical discharges by attenuating its predicted electrosensory reafference ${ }^{21,29}$. In primates, activity in the vestibular nucleus in response to vestibular reafference is attenuated during active head movements compared to passive head movements, allowing the animal to maintain its head and body posture and activate vestibular-related reflexes when appropriate $20,21,30-32$

At the same time, another branch of experimental research has shown that somatosensory sensitivity in response to externally generated stimuli is gated during and before a voluntary movement. In human psychophysical research, this phenomenon of movement-related tactile gating or tactile suppression manifests as an elevation of the detection threshold ${ }^{33-38}$, a decrease in the detection rate ${ }^{33-35,39-43}$, a decrease in the detection precision ${ }^{36,38,44,45}$, and a decrease in the subjective intensity of externally generated stimuli ${ }^{35,40,46}$ when the stimulated body part moves compared to when it is at rest. Several electrophysiological studies have shown that this gating reflects inhibition of somatosensory evoked potentials during active movement compared to rest at subcortical and cortical sites along the somatosensory pathway ${ }^{46-51}$. Similar to somatosensory attenuation, tactile gating is a biologically preserved mechanism that is seen across different species ${ }^{52}$. For example, responses recorded at the cat's medial lemniscus evoked by nerve stimulation are suppressed prior to and during limb movements ${ }^{53}$. Similarly, the transmission of cutaneous afferent signals to the primary somatosensory cortex is suppressed in rats during movement compared to rest ${ }^{54}$. In monkeys, the gating of cutaneous afferent input during active movement has been observed in both the primary somatosensory cortex ${ }^{55-57}$ and the spinal cord ${ }^{57,58}$.

Somatosensory attenuation and tactile gating share two important conceptual similarities. First, they both refer to modulation, either in terms of magnitude or precision, of the perception of cutaneous stimuli during movement. Second, they have been assigned the same functional role ${ }^{41}$ : to reduce the flow of afferent information that can be predicted from the motor command so that detection of external input that can be biologically important, such as touches caused by predators ${ }^{17,24,59}$, or input that is task-relevant for the upcoming or ongoing movement ${ }^{48,50,60}$ is facilitated.

Importantly, however, the two phenomena present one striking difference. Somatosensory attenuation relates to somatosensory reafference, that is, touches caused by our voluntary movement. In contrast, gating relates to somatosensory exafference, that is, external touches occurring during our voluntary movement. Nevertheless, somatosensory research, including very recent studies and review articles, e.g., ${ }^{36,37,61-67}$, often treats the two phenomena as a single generalized suppression strategy of the brain and uses the terms attenuation and gating interchangeably. If the two phenomena are indeed different, this false equivalence is detrimental for understanding human sensorimotor control. First, it prevents advancements in our understanding at the computational level because researchers try to explain gating (e.g., ${ }^{36}$ ) and attenuation (e.g., ${ }^{68}$ ) using the computational processes proposed for attenuation and gating, 
respectively. Similarly, at the neurobiological level, researchers intermix neural correlates of gating and attenuation (e.g., ${ }^{65,66}$ ) because they assume that these measure the same single phenomenon. Second, this confusion becomes particularly disadvantageous in clinical studies using gating and attenuation when interpreting findings of sensorimotor deficits in patients with schizophrenia ${ }^{69-71}$, functional movement disorders ${ }^{72}$ or Parkinson's disease ${ }^{48,73,74}$.

Are these phenomena the same, or do they represent two fundamentally distinct processes? Does the brain treat all sensory stimuli similarly during movement, independent of whether they are reafferent or exafferent? To the best of our knowledge, there has been no previous attempt to simultaneously test attenuation and gating with the same stimulus and psychophysics task. Here, in a single experimental design, we investigated the perception of touches applied on the left hand while manipulating whether the left arm was in movement or resting (left limb state). We additionally manipulated whether the touches were reafferent, generated by the right hand, or exafferent, generated by an external source (origin of touch). We reasoned that if the two phenomena are the same, they should influence somatosensory perception in similar ways. Our results demonstrate that this is not the case: voluntary movement reduces the somatosensory precision of both sensory reafference and exafference (gating), while the perceived amplitude of sensory reafference is selectively attenuated compared to that of exafference (attenuation). Notably, the two phenomena do not correlate with each other or interact when present simultaneously. Thus, collectively, our results show that gating and attenuation are two separate processes that can be experimentally dissociated.

\section{Results}

Participants rested their left hands palm up with their index fingers on a molded support, and their right hands were placed palm down on top of a set of sponges (Fig. 1a-d). In all conditions, they performed a force-discrimination task ${ }^{3,4,10,70}$ : in each trial, a motor delivered two taps (the test tap and the comparison tap) on the pulp of their left index finger, and they were asked to verbally indicate which tap felt stronger (Fig. 1e-h). While the test tap had a fixed intensity (2 $\mathrm{N})$, the intensity of the comparison tap randomly changed in every trial $(1,1.5,1.75,2,2.25$, 2.5 , or $3 \mathrm{~N}$ ). An auditory 'go' signal indicated the trial's onset and the onset of the response period.

In all conditions, our experimental manipulation exclusively concerned the test tap; the comparison tap was always externally triggered and delivered on the relaxed left arm, serving, therefore, as a reference stimulus. In a factorial design, we controlled for whether the left arm moved (Fig. 1c, d) or remained relaxed (Fig. 1a, b) during the test tap and whether the test tap was produced by the right hand (self-generated) (Fig. 1b, d) or not (externally generated) (Fig. 1a, c). This design resulted in four experimental conditions, the order of which was fully counterbalanced (Fig. 1a-d).

In the baseline condition (Fig. 1a), participants did not move their limbs but passively received the test and the comparison taps on the left index. This control condition was used to assess the participants' somatosensory perception in the absence of any movement ${ }^{2,4,10}$. In the attenuation condition (Fig. 1b), participants actively tapped with their right index finger a force sensor placed on top of their left index finger. The tap of their right index finger on the force sensor triggered the test tap on their left index finger. This classic condition was used to assess the perception of a self-generated tap on a passive limb ${ }^{2,4,10}$. In the gating condition (Fig. 1c), participants were asked to continuously move their left arm forward, sliding the experimental setup with the motor and the force sensors between a start and an end position (distance $25 \mathrm{~cm}$ ) 
at a comfortable velocity of approximately $20 \mathrm{~cm} / \mathrm{sec}$ (Fig. 1i-j, Supplementary Fig. S1, S2). During this movement, participants received the test tap on their left index finger. This is a gating condition because it assesses the perception of an externally generated tap on a moving limb $39,40,42,75$. Finally, in the attenuation\&gating condition (Fig. 1d), the participants performed the same movement with their left arm, but they were additionally asked to actively tap with their right index finger on the force sensor that triggered the test tap on their left index finger during the movement. The force sensor was attached to the experimental setup and moved together with the left hand. This condition combines the gating and attenuation phenomena since it is used to assess the perception of a self-generated tap on a moving limb.

a

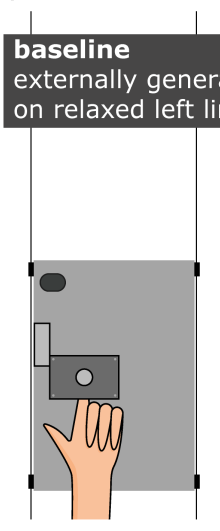

e

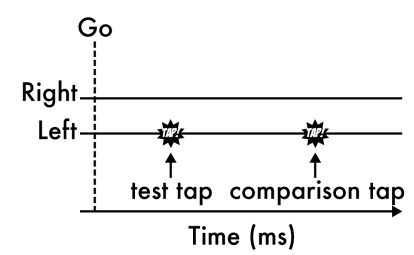

i

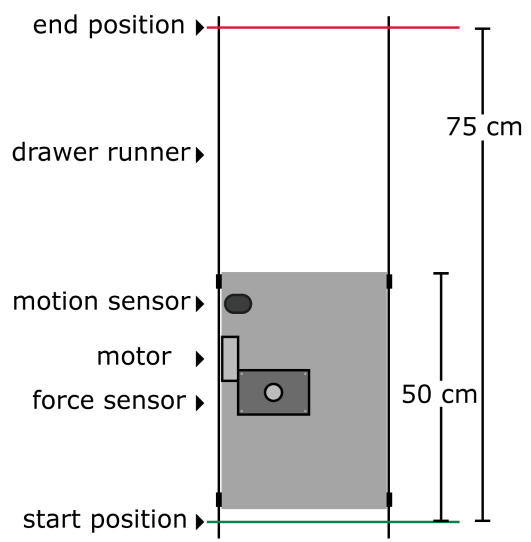

b

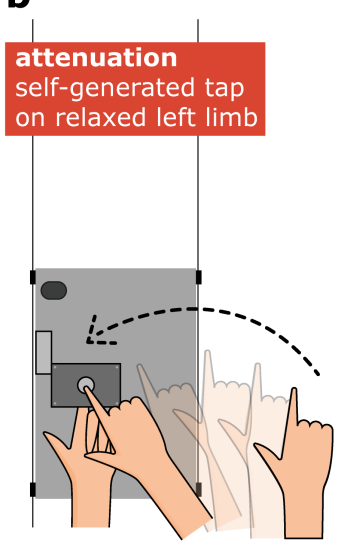

$\mathbf{f}$

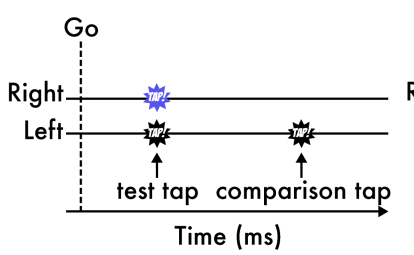

j
C

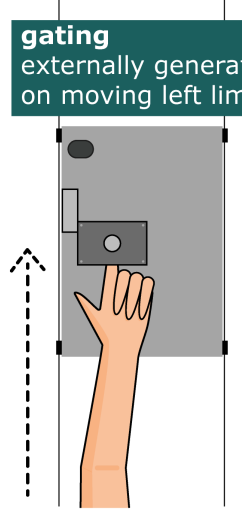

g

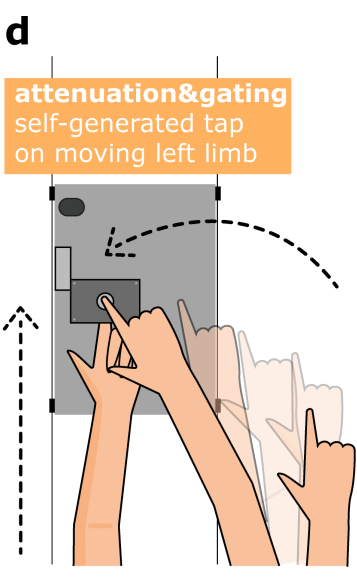

h

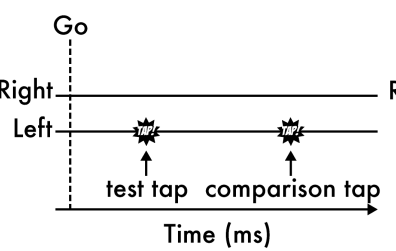

k

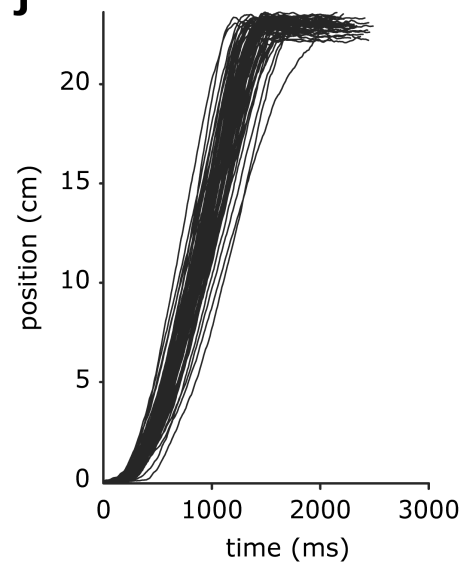

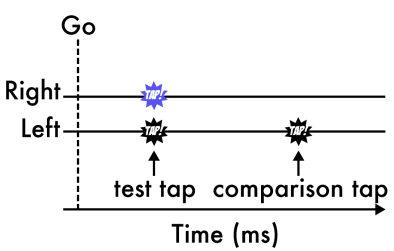
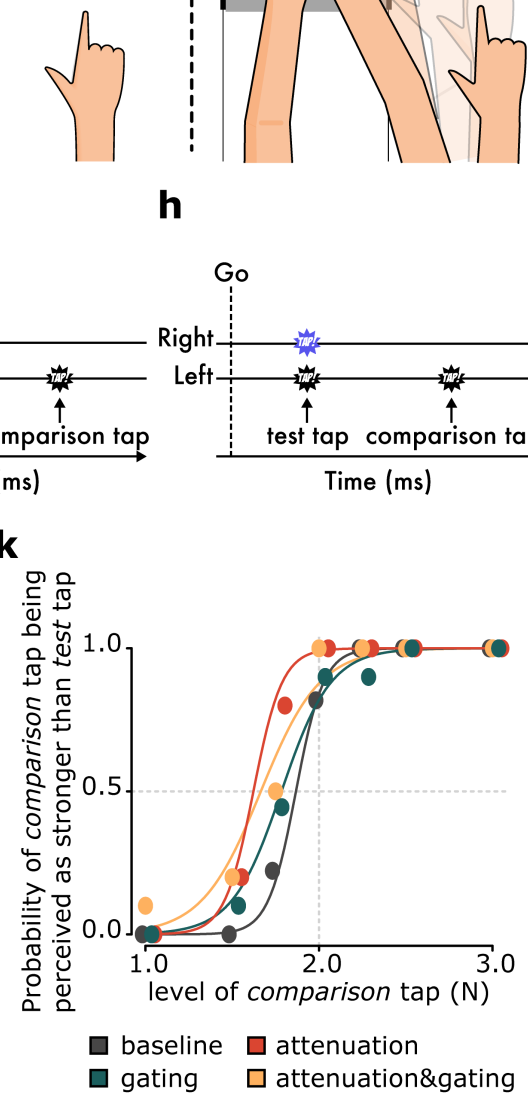

160

Fig. 1. Experimental conditions. Two factors were manipulated in the experiment, resulting in four experimental conditions: whether the left arm was in rest $(\mathbf{a}, \mathbf{b})$ or in movement while receiving the test tap (c, d) (state of the left limb) and whether the test tap was externally triggered by the motor (a, c) or self-triggered by the participants' right hand (b, d) (origin of touch). In all four conditions (e-h), the participants received the two taps (test and comparison tap) on the pulp of their left index fingers from the motor (black stars), and they had to verbally indicate which was stronger: the first or the second tap. In the attenuation and attenuation\&gating conditions, the participants self-triggered the test tap on their left index finger by moving their right arm to tap a sensor with their right index finger (blue stars). (i) In the gating and attenuation\&gating conditions ( $\mathbf{c}$ and $\mathbf{d}$ ), the participants extended their left arm from a starting position to an ending position, sliding the experimental setup along two drawer runners. 
171 During this movement, participants experienced the test tap. A motion sensor recorded the position of the platform in time. (f) Example position traces recorded by the motion sensor for the movements of one participant during the attenuation\&gating condition. (g) Responses of a representative participant and fitted logistic models for the four experimental conditions. The data points were horizontally jittered to avoid overlapping.

The participant's responses in each condition were fitted with a generalized linear model (Fig. $1 \mathbf{k}$, Supplementary Fig. S3), and two parameters of interest were extracted: the point of subjective equality (PSE), which represents the intensity at which the test tap felt as strong as the comparison tap $(p=0.5)$, and the just noticeable difference (JND), which reflects the participants' sensitivity in force discrimination. A lower PSE in an experimental condition indicates that the test tap felt weaker in that condition. A higher JND in an experimental condition indicates that the discrimination sensitivity was lower in that condition (i.e., a larger difference in the force intensities needed to be detected). We hypothesized that the two phenomena are different and, thus, that they affect the PSE and JND differently. Specifically, we expected a decrease in the perceived magnitude (lower PSE) for conditions with sensory reafference (attenuation and attenuation\&gating conditions), with no effects on the sensory precision of the participants (JND) when they received reafference on a still limb (attenuation condition). That is, a self-generated touch will feel weaker than an externally generated touch, but there will be no effect on somatosensory precision. In contrast, we predicted a decrease in the somatosensory precision (higher JND) of both sensory reafference and exafference for conditions where the limb that receives the touches moves (gating, attenuation\&gating). That is, the precision with which a touch (either self-generated or externally generated) is perceived is lower on a moving limb because of the additional kinaesthetic, proprioceptive, tactile signals from the movement. A small, if any, effect in the PSE was expected for the gating condition. We hypothesized that the attenuation phenomenon mainly affects the PSE and not the JND, and the gating phenomenon mainly affects the JND and not the PSE. Our hypotheses were supported by the data (Fig. 2a-g). 

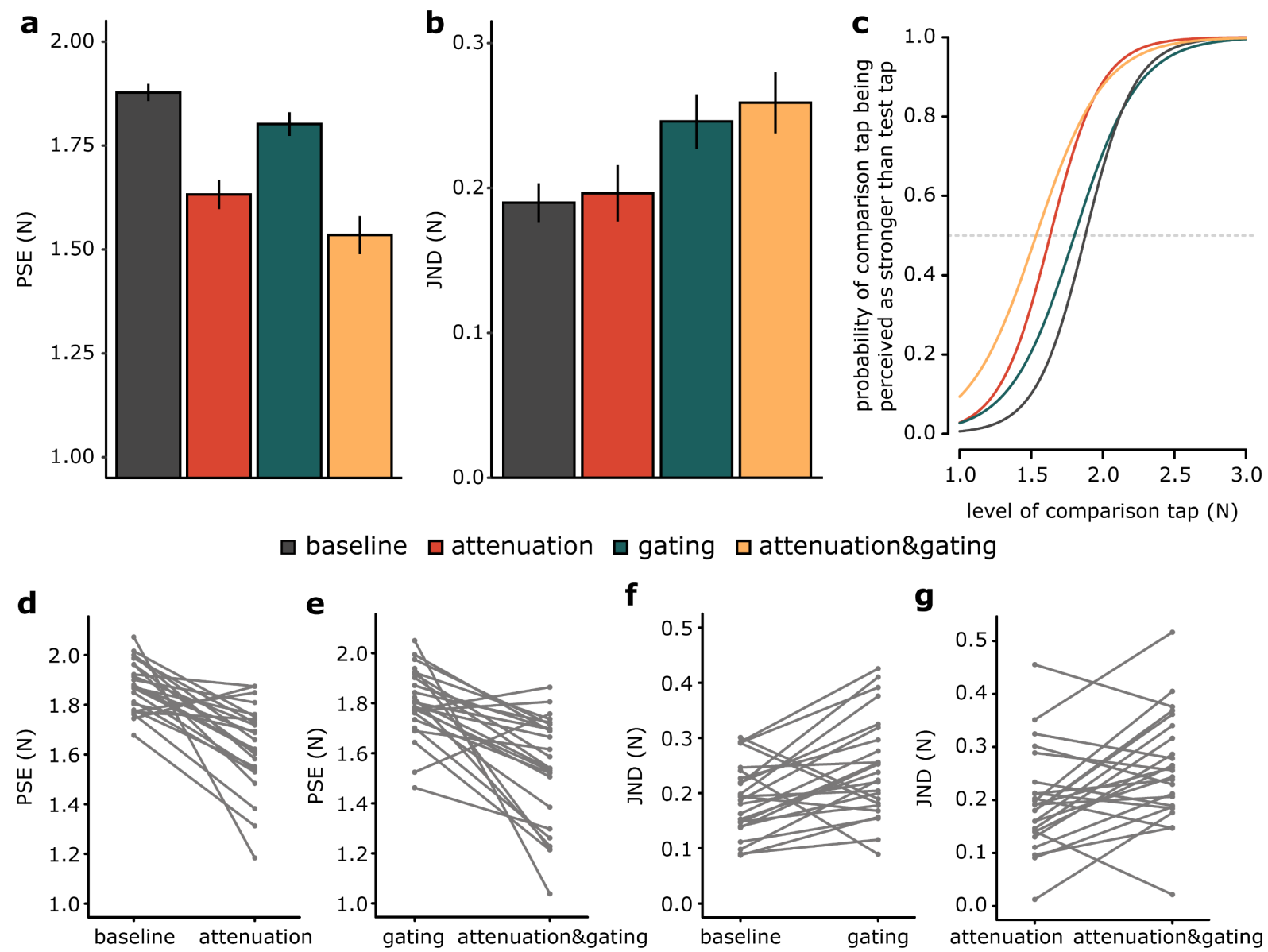

Fig. 2. Experimental results. (a, b) Bar graphs showing the PSEs and JNDs (mean \pm SEM) for each condition. A lower PSE value indicates a lower perceived magnitude, while a higher JND value indicates lower somatosensory sensory precision. Reafferent touches produced by the movement of the right arm (attenuation and attenuation\&gating conditions) were associated with a significant drop in the PSEs, while movement of the left arm that receives the touches (gating and attenuation\&gating) results in a significant increase in the JNDs. A small decrease in PSE was also observed in the gating and attenuation\&gating conditions compared to the baseline and attenuation conditions, respectively. (c) Group psychometric functions for each condition generated using the mean PSE and the mean JND across participants. The leftward shift of the curves for the attenuation and attenuation\&gating conditions illustrates that somatosensory reafference is perceived as weaker than the exafference. The flattening of the curves for the gating and attenuation\&gating conditions illustrates the worsening in somatosensory precision of both the reafference and exafference on a moving limb. (d, e) Line plots illustrating the decreases in PSEs when experiencing reafferent touches compared to exafferent touches when the left arm is still (d) and when the left arm moves (e). (f, g) Line plots illustrating the increases in JNDs when receiving touches on a moving limb compared to rest when touches are exafferent (f) and when touches are reafferent $(\mathrm{g})$.

We performed repeated-measures ANOVA on the PSEs with the origin of the touch (reafference $v s$ exafference) and the state of the left limb (movement $v s$ rest) as factors. This revealed a significant main effect of the origin of the touch $\left(F(1,23)=36.10, p<0.001, \eta_{p}{ }^{2}=\right.$ $0.611)$, a significant main effect of the left limb state $\left(F(1,23)=13.91, p=0.001, \eta_{p}{ }^{2}=0.377\right)$ and a nonsignificant interaction $\left(F(1,23)=0.26, p=0.615, \eta_{p}{ }^{2}=0.011\right)$ (Fig. 2a). A Bayesian repeated-measures ANOVA further concluded against the interaction term by supporting the model without the interaction term compared to the full factorial $\left(B F_{M 1} / B F_{M 2}=3.631\right)$. The attenuation condition produced a significant drop in the PSE compared to the baseline condition $\left(n=24, t(23)=-5.908, p<0.001\right.$, Cohen's $\left.d=-1.206, C I^{95}=[-0.332,-0.160], B F_{01}<0.0003\right)$ 
(Fig. 2d). This replicates previous attenuation findings indicating that a self-generated tap on a passive limb feels weaker than a tap of the same intensity but of an external origin $2,4-7,10,13,16$. Similarly, the PSE in the attenuation\&gating condition was significantly lower than that in the gating condition $\left(n=24, t(23)=-5.032, p<0.001\right.$, Cohen's $d=-1.027, C I^{95}=[-0.377,-0.157]$, $B F_{01}=0.002$ ) (Fig. 2e), extending the previous conclusion to when the receiving limb is moving. Together, these two contrasts show that reafferent (self-generated) touches feel weaker than exafferent touches, both when the receiving hand is in movement and when it is at rest.

The gating and attenuation\&gating conditions also resulted in a significant drop in the PSE compared to that in the baseline condition $(n=24, t(23)=-2.409, p=0.024$, Cohen's $d=-$ $\left.0.492, C I^{95}=[-0.141,-0.011]\right)$ and the attenuation condition $(n=24, V=55, p=0.005, r r b=-$ $\left.0.633, C I^{95}=[-0.161,-0.022]\right)$, respectively. However, these decreases were quite modest $(\cong$ $30 \%$ of the weakening produced by the attenuation condition) and supported only by anecdotal evidence from Bayesian statistics $\left(B F_{01}=0.433\right.$ and $B F_{01}=0.738$, respectively). Together, these contrasts suggest that exafferent touches can feel slightly weaker on a moving limb than on a passive limb, in agreement with previous findings regarding tactile gating $35,40,46$. Nevertheless, when compared with tactile reafference, the perceived magnitude of tactile exafference is not strongly decreased.

When testing for the effects of the conditions on the somatosensory precision of the participants (JND), there was a significant main effect of the state of the left $\operatorname{limb}(F(1,23)=17.1, p<$ $\left.0.001, \eta_{p}{ }^{2}=0.426\right)$, but there was neither a significant main effect of the origin of touch $(F(1$, $\left.23)=0.52, p=0.478, \eta_{p}{ }^{2}=0.022\right)$ nor a significant interaction $\left(F(1,23)=0.06, p=0.809, \eta_{p}{ }^{2}\right.$ $=0.003$ ) (Fig. 2b). As with PSEs, the absence of interaction for the JNDs was further supported by Bayesian repeated-measures ANOVA, which provided evidence against the interaction term $\left(B F_{M 1} / B F_{M 2}=3.522\right)$. The attenuation condition did not result in any change in the JND with respect to that in the baseline condition $(n=24, t(23)=0.331, p=0.744$, Cohen's $d=0.068$, $\left.C I^{95}=[-0.034,0.047]\right)$, and this was substantially confirmed by Bayesian analysis $\left(B F_{01}=\right.$ 4.432). In alignment, no significant differences in the JND were detected between the gating and the attenuation\& gating conditions $\left(n=24, t(23)=0.72, p=0.481\right.$, Cohen's $d=0.146, C I^{95}$ $=[-0.024,0.05])$, which was again confirmed by Bayesian analysis $\left(B F_{01}=3.691\right)$. Together, these two contrasts demonstrate that receiving sensory reafference per se is not accompanied by worsening of sensory precision on the receiving limb.

In contrast, moving the limb while receiving an external touch (gating condition) resulted in a significant increase in the JND compared to that in the baseline condition $(n=24, t(23)=3.134$, $p=0.005$, Cohen's $\left.d=0.640, C I^{95}=[0.019,0.093], B F_{01}=0.108\right)$ (Fig. 2f). This was further confirmed by a significant increase in JND in the attenuation \& gating condition compared with the attenuation condition $\left(n=24, t(23)=2.984, p=0.007\right.$, Cohen's $d=0.609, C I^{95}=[0.019$, 0.106], $B F_{01}=0.146$ ) (Fig. 2g). Together, these two differences indicate that voluntary movement per se deteriorates the precision with which reafferent and exafferent stimuli are perceived on the moving limb.

Together, our results indicate that predicting the sensory consequences of a voluntary movement produces a decrease in the perceived magnitude of sensory reafference (PSE) without a concomitant worsening of somatosensory precision (JND). In contrast, voluntary movement leads to a decrease in somatosensory precision (JND) for both sensory reafference and exafference. These effects are observed in the group psychometric fits (Fig. 2c). To further illustrate that somatosensory attenuation affects the amplitude (PSE), while tactile gating affects the precision (JND) and not vice versa, we tested whether we could better predict the 
participants' performance in the attenuation\&gating condition when using the PSE from the attenuation condition and the JND from the gating condition than the PSE from the gating condition and the JND from the attenuation condition. Indeed, the first model was significantly better: $n=24, V=39, p<0.001, r r b=-0.74, C I^{95}=[-68.980,-12.736], B F_{01}=0.443$ (Supplementary Fig. S4, Fig. 3a-b).

In the abovementioned ANOVAs, there were no significant interactions between the two factors (the origin of touch and the state of the limb), neither for the PSEs nor for the JNDs, according to both frequentist and Bayesian analyses. This indicates that the effect of the left limb state was not influenced by the effect of the origin of touch, neither for the PSE nor for the JND. Instead, the two effects were summed when simultaneously present, and there were no superadditive effects (interactions), which is in line with the proposal that they are two independent processes. To further test whether the effects produced by each phenomenon relate to each other at all, we performed a correlation analysis of the PSEs and JNDs. No significant correlations were detected between any of the PSEs and any of the JNDs (all $p$-values $>0.225$, $\left.B F_{01}=[1.971,3.950]\right)$. The only significant correlation found for PSEs was between the PSE in the attenuation condition and the PSE in the attenuation\& gating condition $(t(22)=4.89, r=$ 0.722, $p<0.001, C I^{95}=[0.449,0.871], B F_{01}=0.002$ ) (Fig. 3c). That is, the weaker the participants perceived the magnitude of their self-generated touch during rest, the weaker the magnitude of their self-generated touch during movement felt. Given that the PSEs significantly dropped in these two conditions and that these decreases correlated with each other, this result provides further support that their common experimental denominator, i.e., the reafferent nature of the touch, was responsible for the drop in the PSE and thus for the attenuation phenomenon. In contrast, the JND in the gating condition was significantly correlated only with the JND in the attenuation \& gating condition $\left(t(22)=3.47, r=0.595, p=0.008, C I^{95}=[0.252,0.805], B F_{01}\right.$ $=0.047)($ Fig. 3d). This specific correlation means that the worse the somatosensory precision of an external touch when participants moved their receiving hand, the worse the sensory precision for a self-generated touch during the same movement of the receiving hand. Given that the JNDs significantly increased only in these two conditions and that these increases correlated with each other, this result provides evidence that their common experimental denominator, i.e., the left limb's movement that receives the touches, was responsible for the increase in the JND. 
a

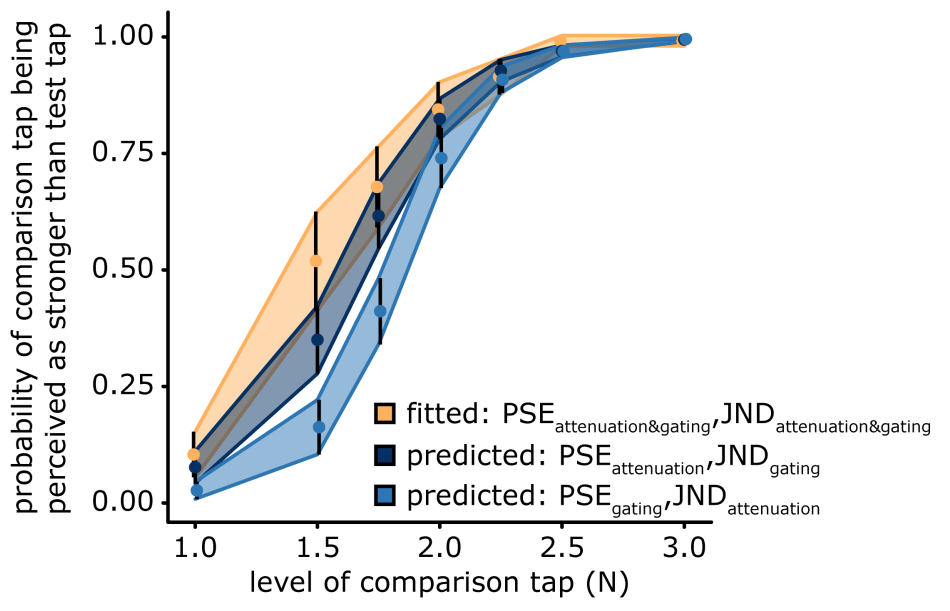

b

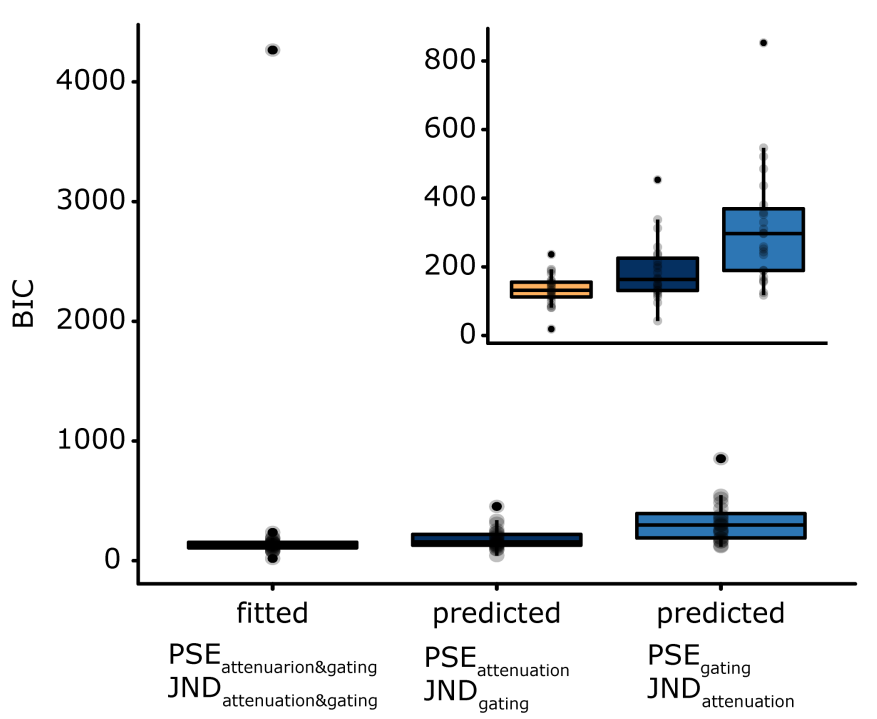

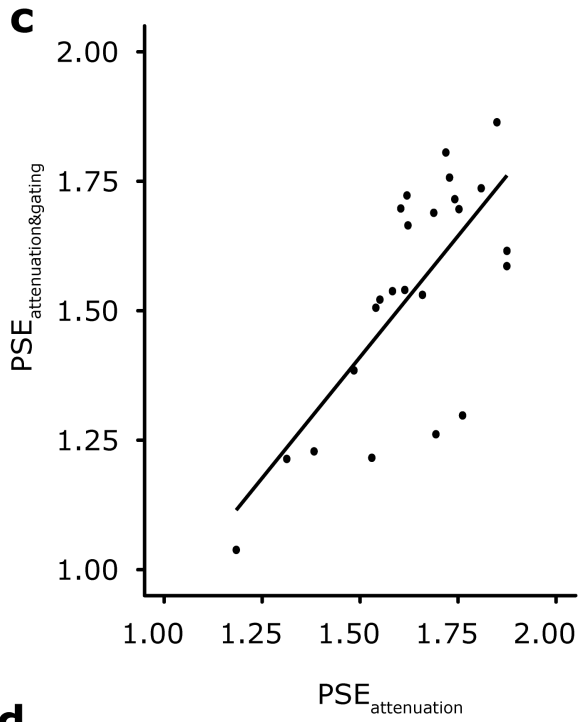

d

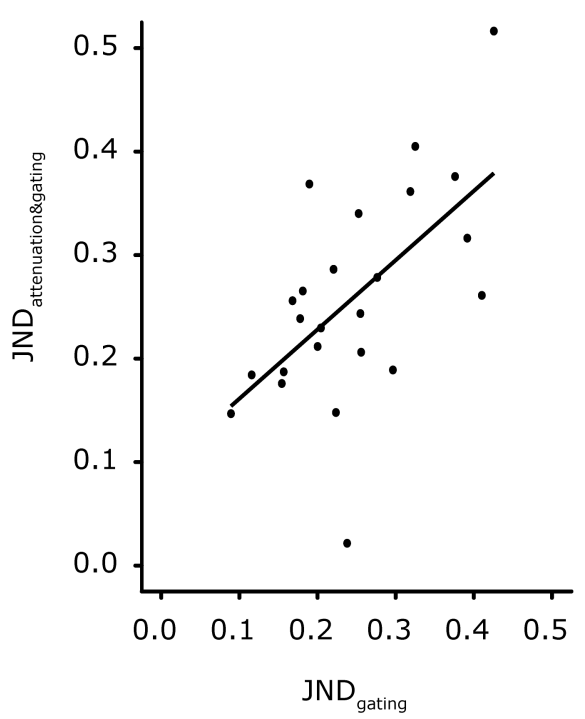

Fig. 3. Model predictions and scatterplots for PSEs and JNDs. (a) Average participants' responses in the attenuation\& gating condition (yellow) and average predicted responses using the parameters from the attenuation and gating conditions (blue). The responses depicted in dark blue were for the PSE of the attenuation condition and the JND from the gating condition, while the responses depicted in light blue were for the PSE of the gating condition and the JND of the attenuation condition. The error bars and ribbons represent $95 \%$ confidence intervals. (b) For each participant, we estimated the Bayesian information criterion (BIC) of the fitted logistic model in the attenuation\&gating condition and the two models with fixed parameters from the attenuation and gating conditions. The model using the PSE of the attenuation condition and the JND of the gating condition was a significantly better model than the one using the PSE of the gating condition and the JND from the attenuation condition. The upper right panel represents the same data after excluding one participant corresponding to the outlier for illustration purposes. The exclusion of the outlier did not change the statistical results. (c) The participants' PSEs in the attenuation condition were significantly correlated with those in the attenuation\&gating condition. (d) The participants' JNDs in the gating condition were significantly correlated with those in the attenuation\&gating condition. There were no significant correlations between PSEs and JNDs.

A common finding in tactile gating studies is that the gating effects, both behavioral and electrophysiological, are stronger with higher movement velocities $33,36,39,42,76$; that is, the faster the limb movements are, the worst the perception of the moving limb. Therefore, one could 
hypothesize that any differences observed between the gating and attenuation\&gating conditions might be due to differences in the velocity of the participants' movements. Since there were no significant JND differences in these two conditions (Fig. 2), which was further supported by Bayesian analysis, this concern can be ruled out. However, one can argue that the PSE in the attenuation\&gating condition was lower than the PSE in the attenuation condition because the participants moved faster in the attenuation\&gating condition and not because of the reafferent nature of the touch. This concern can also be ruled out since, rather than faster, we observed that the participants moved slightly slower in the attenuation\&gating condition $(20.3 \pm 0.003 \mathrm{~cm} / \mathrm{s})$ than in the gating condition $(23.3 \pm 0.003 \mathrm{~cm} / \mathrm{s})$ : (peak trial velocity; $t(23)$ $=-4.062, p<0.001$, Cohen's $\left.d=-0.829, C I^{95}=[-0.001,-0.004], B F_{01}=0.015\right)$. Although the total distances the participants ran with their left arm were comparable, participants in the attenuation\&gating condition moved slower because they had to coordinate both their arms to tap the sensor that the left arm moves with the right hand (Fig. 1d). This difference was further confirmed when looking at the peak velocities during the test tap between the two conditions (gating: $22.2 \pm 0.003 \mathrm{~cm} / \mathrm{s}$; attenuation\&gating: $19 \pm 0.003 \mathrm{~cm} / \mathrm{s})($ Supplementary Fig. S5). Therefore, these kinematic differences cannot explain the significant decrease in the PSEs in the attenuation\&gating condition compared to the gating condition, but they actually

\section{Discussion}

The present study contrasted the conditions of attenuation and gating in a single experimental paradigm to investigate the relationship between the two phenomena. To this end, we independently manipulated the origin of the touch (reafference $v s$ exafference) and the state of the receiving limb (movement vs rest), studying all four possible combinations of these levels. We replicated the classic phenomenon of predictive attenuation of touch ${ }^{1,2,16,3-10}$ by showing that somatosensory reafference feels weaker than somatosensory exafference. Importantly, however, this decrease in the perceived amplitude (PSE) was not accompanied by a concomitant worsening of somatosensory precision (JND). That is, participants had the same discrimination capacity (JND) for both reafferent and exafferent touches applied on their passive limb, a conclusion supported by Bayesian statistics. Nevertheless, when the limb that received the touches moves, this voluntary movement per se led to a decrease in somatosensory precision (JND) for both reafferent and exafferent touches, replicating the classic tactile gating phenomenon ${ }^{33-37,39,41,42}$. The two effects did not correlate and did not interact, but were summed when present together.

The main conclusion of the present study is that the predictive attenuation of touch and tactile gating are two distinct perceptual phenomena. Our findings can help conciliate several previous observations on gating and attenuation that have been studied in isolation during recent decades. First, attenuation is observed not only on the active $\operatorname{limb}{ }^{8}$ but also on a passive limb (the contralateral hand in the present study), as long as the contact between the body parts is predicted by voluntary movement $2-7,9,10,13,16$. In contrast, there is abundant evidence that external touches applied to the limb contralateral to the limb that moves are not gated $34,45,46,48,77,78$. Second, a touch that results from a passive movement ${ }^{10}$ or touches that are simultaneously presented in both hands (double touch) ${ }^{70}$ are not attenuated. In contrast, gating effects have been repeatedly demonstrated for passive movements, both electrophysiologically ${ }^{48}$ and behaviorally ${ }^{34,41,75}$. Third, self-generated tactile signals are attenuated as long as they are presented at the timing predicted by the action ${ }^{1,2,4}$; even a 100 ms delay between the movement and its tactile feedback greatly reduces the attenuation of the latter. In contrast, externally 
generated stimuli are gated with less temporal sensitivity; for example, gating is observed for stimuli presented at several (unpredicted) times during the movement ${ }^{48}$, at the movement onset ${ }^{43}$, and importantly, even at hundreds of milliseconds before the movement onset $39,41,43,45$. Fourth, whereas gating shows no specificity for the type of motor activity and manifests both during isotonic ${ }^{33-35,46}$ and isometric ${ }^{35,78}$ contractions, attenuation is motor command-specific; a consistent but arbitrary and unnatural mapping between the motor command and the touch, for example, moving a joystick with one hand to produce touch on the other, does not produce attenuation $5,6,72$. These results are not contradictory in light of our findings; instead, they refer to different perceptual phenomena.

Our study is the first to behaviorally dissociate the predictive attenuation of touch and tactile gating. A previous study ${ }^{79}$ found that the perceived intensity of an electrical stimulus applied to the tip of the relaxed left index finger does not change if this finger receives a self-produced force generated by the right index finger. However, in that study, the perception of touch was tested on a resting limb only, which is already known not to elicit gating effects ${ }^{34,45,46,48,77,78}$. Therefore, the two phenomena were not disentangled because they were not tested on the same limb as in the current study. Similarly, another study ${ }^{65}$ measured electrophysiological responses (somatosensory evoked potentials) to external electrical stimulation on the right and the left wrist, while the participants produced a self-generated force with their right index finger on their left index finger. The authors did not measure the perception of external touches during movement (gating) but only the perception of self-generated touches during rest (attenuation) and could thus not dissociate the two phenomena behaviorally. Moreover, the authors observed modulation of the late electrophysiological responses depending on whether the participants produced a self-generated force, and they suggested that the attenuation and gating might share the same mechanism: that of reduced sensory precision. However, the present study demonstrates that sensory precision is reduced during gating and not during attenuation, suggesting that a common mechanism governing both phenomena is unlikely.

Motor control relies on integrating afferent sensory information with efferent motor signals ${ }^{79}$. Distinguishing between gating and attenuation is fundamentally important for motor control theories because it can indicate a different integration or weighting mechanism of the motor and sensory information depending on the context. One well-established computational framework inspired by engineering approaches poses that the brain produces motor commands through an inverse model ${ }^{80}$ or controller ${ }^{81}$. A copy of the motor command, termed 'efference copy', is used by a forward model to predict the expected sensory feedback of the movement, which is then combined with the actual sensory input to estimate the state of the body ${ }^{59,80-82}$. With respect to the attenuation of sensory reafference, it has been proposed that the prediction signal of the forward model is used to 'cancel' the sensory reafference ${ }^{17,59,83-85}$. In other words, central motor processes play a more important role in somatosensory attenuation than actual sensory feedback. Support for this comes from studies showing that conditions that present highly predictable touches but in the absence of movement do not yield attenuation ${ }^{2,10}$ (see also 86-88 for similar conclusions). The dependence of attenuation on action prediction was further shown when participants attenuated the touches applied to one hand that were predicted by their other hand's movement, even when the two hands unexpectedly failed to make contact ${ }^{3}$. In further agreement, neuroimaging studies on somatosensory attenuation consistently report activation of the cerebellum ${ }^{11-15}$, a structure that is associated with motor prediction ${ }^{79,89-92}$. Our findings of a reduced perceived magnitude of somatosensory reafference compared to exafference (i.e., lower PSEs in the attenuation and attenuation\&gating conditions compared to the baseline and gating conditions, respectively) support this forward model mechanism for the attenuation of self-generated input. 
In contrast, this computational account that relies on action prediction, efference copy, and internal forward models is inapplicable for tactile gating since gated touches can be of exafferent nature and occur at any (unpredictable) time during movement, even before movement onset $39,40,50,75$. In other words, there is no information that the brain can use to predict exafferent touches with the forward model because there is no causal relationship between the motor command and sensory input. This observation agrees with the proposal that peripheral afferent signals from muscle spindles and joint afferents play the major role in gating ${ }^{39,48}$ and that gating effects have also been observed during passive movement, without significant differences from active movements ${ }^{34,75}$. Then, if efference copy is not the basis for gating, how can the gating effects be computationally explained?

The alternative computational framework of active inference has been proposed to explain somatosensory attenuation ${ }^{62}$; however, this was based on an assumed equivalence between attenuation and gating. The active inference approach refutes the necessity of an efference copy and emphasizes the importance of a generative model and reflex arcs in the place of forward and inverse models and controllers ${ }^{93,94}$. According to the active inference account, the brain predicts the sensory input that would be expected from a specific action, and the body moves to fulfill these sensory predictions. Motor commands are thus conceptually replaced by proprioceptive predictions, and action occurs as a way to minimize the proprioceptive prediction errors when the movement has not yet been executed ${ }^{95}$. A major role is assigned to the precision (i.e., reliability), which weights these sensory prediction errors depending on the context and can be manipulated through attention allocation. Within this computational architecture, attenuation of somatosensory reafference is seen as a reduction in the precision of somatosensory evidence during movement to allow the expression of proprioceptive predictions that trigger the movement ${ }^{62}$. In other words, the agent attends away from somatosensory input to execute the movement. However, this proposal does not address the attenuation of sensory reafference with respect to exafference since the agent should theoretically attend away from all somatosensory inputs independent of their source. Moreover, the proposal cannot explain why increasing the precision of the proprioceptive prediction errors on the hand that is to move reduces the precision of somatosensory prediction errors on the contralateral limb that is not meant to move and thus, there should not exist any proprioceptive predictions about it. In contrast, the active inference account can sufficiently explain the tactile gating effect, i.e., the reduction in the precision of somatosensory input on the moving limb during movement. Our findings of a reduced precision of somatosensory input on the moving limb (i.e., higher JNDs in the gating and attenuation\&gating conditions compared to the baseline and attenuation conditions, respectively) are compatible with this active inference mechanism for the gating of sensory input during movement.

Another mechanism that has been proposed to explain tactile gating is a backward masking or postdictive mechanism ${ }^{68}$ that is not necessarily dependent on motor signals. Accordingly, when moving a limb, the sensation from the muscles, joints, and skin of the moving limb masks the externally generated touches that are applied on this limb. These sensations could affect the perception of earlier stimuli in a postdictive manner ${ }^{75}$, and this could explain the gating effects observed for external touches applied even before the movement onset ${ }^{41}$. Our gating findings of a reduced precision of somatosensory input on the moving limb are compatible with this backward masking mechanism. Furthermore, in the context of a passive movement (i.e., in the absence of motor commands), the sensations from the muscles, joints, and skin of the passively moving limb could mask the externally generated touches on the same limb, and this 
mechanism could account for the absence of gating differences between active and passive movements ${ }^{34,75}$.

Finally, a recent study ${ }^{67}$ suggested that action prediction results in somatosensory enhancement rather than attenuation and argued that the attenuation observed in all earlier experiments is actually due to nonpredictive gating processes. This claim cannot be supported based on several observations and arguments. First, the present results showed that the predictive attenuation of touch and gating are different phenomena; if sensory attenuation was the same as tactile gating, then all stimuli applied on the moving limb would be attenuated. In contrast, we show that sensory reafference is selectively attenuated compared to sensory exafference, both in passive and moving limbs. Similar results were shown in ${ }^{84}$, where participants attenuated only their reafferent touches and not exafferent touches presented on the same limb simultaneously. Second, Thomas and colleagues ${ }^{67}$ provided participants with an arbitrary mapping between the movement of one hand and sensory feedback on the other hand. Using a very similar setup, a study from $2006^{3}$ found neither attenuation, which is consistent with the predictive account of attenuation, nor enhancement effects, which is inconsistent with the findings of Thomas and colleagues ${ }^{67}$. Third, it is essential to keep in mind that attenuation has also been shown on an anesthetized limb ${ }^{8}$ and for imagined movements ${ }^{9}$, further emphasizing the contribution of motor prediction in relation to peripheral feedback for somatosensory attenuation. Finally, it has been shown that when reaching with one arm towards the other, the perceived intensity of an external tactile stimulus applied to the passive target limb is enhanced ${ }^{44}$. Although we believe that future experimentation is needed to understand and replicate the reported enhancement effects ${ }^{67}$, we speculate that, as in ${ }^{44}$, increased spatial attention or other kinematic differences play a significant role in the somatosensory enhancement reported by Thomas and colleagues ${ }^{67}$.

We conclude that the human brain uses two different basic processes to suppress reafferent and exafferent information during movement and rest. This separation of attenuation and gating can explain why although we cannot tickle ourselves because we attenuate our self-tickles, we do tense our muscles when being tickled by others to decrease our sensitivity to external tickles.

\section{Materials and Methods}

\section{Participants}

After providing written informed consent, twenty-four participants (12 women and 12 men, 22 right-handed, 1 ambidextrous, 1 left-handed) aged 21-40 years participated. Handedness was assessed using the Edinburgh Handedness Inventory ${ }^{96}$. The sample size was set to twenty-four (24) before data collection commenced based on our previous studies using the same methods 4,10 while ensuring a counterbalanced order of conditions. Three participants were excluded because of technical issues with the kinematic recordings and replaced by three new participants to reach the target sample size. The Swedish Ethical Review Authority (https://etikprovningsmyndigheten.se/) approved the study (no. 2016/445- 31/2, amendment 2019-04536). All participants provided their written informed consent.

\section{General Procedure}

Participants sat comfortably on a chair with their arms placed on a table. Their left hands rested palm up, with their index fingers placed on a molded support. The right arms rested palm down on top of a set of sponges. In each trial, a motor (Maxon EC Motor EC 90 flat; Switzerland) delivered two taps (the test tap and the comparison tap) on the pulp of their left index finger through a cylindrical probe $(25 \mathrm{~mm}$ height $)$ with a flat aluminum surface (20 mm diameter) 
attached to the motor's lever. A force sensor (FSG15N1A, Honeywell Inc.; diameter, $5 \mathrm{~mm}$; minimum resolution, $0.01 \mathrm{~N}$; response time, $1 \mathrm{~ms}$; measurement range, $0-15 \mathrm{~N}$ ) within the probe recorded the forces applied on the left index finger. Following the presentation of the two taps, participants had to verbally indicate which tap felt stronger: the first or the second. A second identical force sensor within an identical cylindrical probe was placed on top of, but not in contact with, the probe of the left index finger (Fig. 1).

A wooden surface was placed under the motor and the sensors. This surface was placed on top of two commercially available drawer runners (IKEA, https:/www.ikea.com/us/en/p/bestadrawer-runner-soft-closing-40348715/). One side of the runners was attached to the table with Velcro, and the other side was attached to the bottom side of the surface. With this configuration, the surface, with the motor, the sensors and the participants' hands, could be moved forward and backward.

In all conditions, the comparison tap was delivered on the left index finger with a random delay of 800-1500 ms from the test tap. In the attenuation and attenuation\&gating conditions (Fig. 1b, d), the tap of the participants' right index fingers on the force sensor triggered the test tap on their left index finger with an intrinsic delay of $\approx 36 \mathrm{~ms}$.

In the gating and attenuation\& gating conditions (Fig. 1c, d), participants were asked to extend their elbow upon an auditory 'go' cue. The extension of the elbow moved the platform forward on the table (Fig. 1f). A piece of green tape on the table (Fig. 1i) indicated the start position of the platform, while a piece of red tape indicated the end position. The participants were asked to move the platform from the start position to the end position (distance $=25 \mathrm{~cm}$ ). During the movement of the left arm, the participants received the test tap on their left index finger. Before the condition started, we emphasized to the subjects that their task was to pay attention to the force that they would receive during the movement rather than covering exactly the distance between the lines. Moreover, the participants were trained to perform the movement in approximately 1000-1500 ms after the 'go' cue and then stop. In the gating condition, the test tap was applied $800 \mathrm{~ms}$ after the 'go' cue so that its delivery was during the movement. Similarly, in the attenuation\&gating condition, the participants triggered the test tap during the movement. The comparison tap was applied 800-1500 ms after the test tap to ensure that the participants had stopped moving. Once the participants gave a response, they returned the platform to the start position.

A motion tracking sensor (6DOF Polhemus Fastrak, USA, weight $=9.1 \mathrm{~g}$, dimensions $=2.29$ $\mathrm{cm} \times 2.82 \mathrm{~cm} \times 1.52 \mathrm{~cm}$ ) was placed on top of the platform to record the motion of the platform due to the movement of the participants' left arm. The sensor recorded the $\mathrm{x}, \mathrm{y}$ and $\mathrm{z}$ positions at a sampling rate of approximately $120 \mathrm{~Hz}$.

Each condition included 70 trials. The test tap was set to $2 \mathrm{~N}$, while the intensity of the comparison tap was systematically varied among seven different force levels $(1,1.5,1.75,2$, $2.25,2.5$ or $3 \mathrm{~N}$ ). Each tap lasted for $100 \mathrm{~ms}$. In every trial, participants verbally indicated which tap on their left index finger felt stronger: the first (test) or the second (comparison). Participants were told not to try to balance their responses (50\% first and 50\% second), and they were further instructed to make their best guess if the intensity of the two taps felt similar.

In addition, participants were administered white noise through a pair of headphones to preclude any sounds created by the motor to serve as a cue for the task. The loudness of the white noise was adjusted so that participants could clearly hear the auditory cues of the trial. In 
all conditions, the view of the pulp of the left index finger was occluded. Participants were asked to fixate on a cross placed on a wall $2 \mathrm{~m}$ opposite them, but they were allowed to look at the force sensor to guide the movement of the right index finger when needed (Fig. 1b, d). No feedback was provided to the participants about their responses.

\section{Force discrimination analysis}

In each condition, the participants' responses were fitted with a generalized linear model using

$$
p=\frac{e^{\beta 0+\beta 1 x}}{1+e^{\beta 0+\beta 1 x}}(\text { Equation } 1)
$$

We extracted two parameters of interest: the PSE, which represents the intensity at which the test tap felt as strong as the comparison tap $(p=0.5)$ and which quantifies the perceived intensity, and the JND, which reflects the participants' discrimination capacity. Before fitting the responses, the values of the applied comparison taps were binned to the closest value with respect to their theoretical values $(1,1.5,1.75,2,2.25,2.5$ or $3 \mathrm{~N})$.

\section{Kinematic analysis.}

Both position and velocity data were smoothed with a moving average filter in MATLAB 2018a. Velocity was calculated as the first derivative of position. To calculate the distance participants ran in every trial of each condition, we calculated the minimum and the maximum position of the platform during the entire trial duration. The peak trial velocity was defined as the peak velocity of the entire trial. The peak tap velocity was defined as the peak velocity during the period that the test tap was applied.

\section{Rejection of trials}

After data collection, one hundred seventy-three (173) trials out of $6720(2.57 \%)$ were rejected. First, in thirty-four trials (34), the intensity of the test tap (2 N) was not applied accurately (test $\operatorname{tap}<1.85 \mathrm{~N}$ or test tap $>2.15 \mathrm{~N}$ ), and in sixteen (16) trials, the responses were missing. Second, we rejected one hundred seventeen (117) trials in total from the gating and attenuation\&gating conditions because participants either did not move their left arm (or moved it too slowly) during the test tap (mean velocity $<10 \mathrm{~cm} / \mathrm{s}$ ) or they moved it during the comparison tap (mean velocity $>5 \mathrm{~cm} / \mathrm{s}$ ). The thresholds were based on a previous study ${ }^{42}$ showing no gating effects for velocities smaller than $5 \mathrm{~cm} / \mathrm{s}$. The analysis was therefore performed with 6547 trials in total.

\section{Statistical analysis}

We used $\mathrm{R}^{97}$ and JASP ${ }^{98}$ to analyze our data. The data normality was assessed using the Shapiro-Wilk test. Depending on the data normality, we then performed planned comparisons using either a paired t-test or a Wilcoxon signed-rank test. We report $95 \%$ confidence intervals $\left(C I^{95}\right)$ for each statistical test. Effect sizes are given by the partial eta-squared $\left(\eta_{p}{ }^{2}\right)$ for the ANOVAs, by Cohen's $d$ for t-tests or by the matched rank biserial correlation $r r b$ for the Wilcoxon signed-rank tests. In addition, a Bayesian factor analysis using default Cauchy priors with a scale of 0.707 was carried out for all statistical tests to provide information about the level of support for the null hypothesis compared to the alternative hypothesis $\left(B F_{01}\right)$ given the data. Finally, correlations were performed using Pearson's coefficient $r$ given that the data were normally distributed. All tests were two-tailed.

\section{Corrections for multiple comparisons}

Since our PSE and JND comparisons were planned, we did not apply corrections for multiple comparisons. However, all results remained exactly the same when applying corrections for the 
false discovery rate (FDR) ${ }^{99}$. In the correlation analyses, we did apply corrections for multiple comparisons (FDR) since, although we expected correlations between the PSEs and between the JNDs, we had no a priori hypotheses for correlations between PSEs and JNDs.

\section{Acknowledgments}

We thank M. Chancel, M. Hauser and L. Miller for their feedback on an earlier version of the manuscript. K.K. was supported by the Swedish Research Council (VR Starting Grant 201901909 granted to K.K.). H.H.E. and experimental costs were supported by the Swedish Research Council, Torsten Söderbergs Stiftelse, and Göran Gustafssons Stiftelse. We thank Kanaka Raghavan for help with the illustrations.

\section{Data availability}

We do not have an ethical permit to make the data publicly available. However, the data that support the findings of this study are available from the corresponding author upon reasonable request.

\section{Conflicts of interest/competing interests}

The authors declare no competing financial interests.

\section{Authors' contributions}

K.K. and H.H.E. conceived and designed the experiment. K.K. collected the data and conducted the statistical analysis. K.K. and H.H.E. wrote the manuscript.

\section{References}

1. Blakemore, S. J., Frith, C. D. \& Wolpert, D. M. Spatio-temporal prediction modulates the perception of self-produced stimuli. J. Cogn. Neurosci. 11, 551-559 (1999).

2. Bays, P. M., Wolpert, D. M. \& Flanagan, J. R. Perception of the consequences of selfaction is temporally tuned and event driven. Curr. Biol. 15, 1125-1128 (2005).

3. Bays, P. M., Flanagan, J. R. \& Wolpert, D. M. Attenuation of self-generated tactile sensations is predictive, not postdictive. PLoS Biol. 4, 281-284 (2006).

4. Kilteni, K., Houborg, C. \& Ehrsson, H. H. Rapid learning and unlearning of predicted sensory delays in self-generated touch. Elife 8, 1-17 (2019).

5. Shergill, S. S., Bays, P. M., Frith, C. D. \& Wolpert, D. M. Two eyes for an eye: the neuroscience of force escalation. Science 301, 187 (2003).

6. Kilteni, K. \& Ehrsson, H. H. Sensorimotor predictions and tool use: Hand-held tools attenuate self-touch. Cognition 165, 1-9 (2017).

7. Kilteni, K. \& Ehrsson, H. H. Body ownership determines the attenuation of selfgenerated tactile sensations. Proc. Natl. Acad. Sci. 114, 8426-8431 (2017).

8. Walsh, L. D., Taylor, J. L. \& Gandevia, S. C. Overestimation of force during matching of externally generated forces. J. Physiol. 589, 547-557 (2011).

9. Kilteni, K., Andersson, B. J., Houborg, C. \& Ehrsson, H. H. Motor imagery involves predicting the sensory consequences of the imagined movement. Nat. Commun. 9 , 1617 (2018).

10. Kilteni, K., Engeler, P. \& Ehrsson, H. H. Efference Copy Is Necessary for the Attenuation of Self-Generated Touch. iScience 23, 100843 (2020).

11. Blakemore, S.-J., Wolpert, D. M. \& Frith, C. D. Central cancellation of self-produced tickle sensation. Nat. Neurosci. 1, 635-640 (1998).

12. Shergill, S. S. et al. Modulation of somatosensory processing by action. Neuroimage 70, 356-362 (2013).

13. Kilteni, K. \& Ehrsson, H. H. Functional Connectivity between the Cerebellum and 
Somatosensory Areas Implements the Attenuation of Self-Generated Touch. $J$. Neurosci. 40, 894-906 (2020).

14. Blakemore, S. J., Frith, C. D. \& Wolpert, D. M. The cerebellum is involved in predicting the sensory consequences of action. Neuroreport 12, 1879-1884 (2001).

15. Blakemore, S. J., Wolpert, D. M. \& Frith, C. D. The cerebellum contributes to somatosensory cortical activity during self-produced tactile stimulation. Neuroimage 10, 448-459 (1999).

16. Wolpe, N. et al. Ageing increases reliance on sensorimotor prediction through structural and functional differences in frontostriatal circuits. Nat. Commun. 7, 13034 (2016).

17. Blakemore, S.-J., Wolpert, D. \& Frith, C. Why can't you tickle yourself? Neuroreport 11, R11-R16 (2000).

18. Leavens, D. A. \& Bard, K. A. Tickling. Curr. Biol. 26, R91-R93 (2016).

19. Weiskrantz, L., Elliott, J. \& Darlington, C. Preliminary observations on tickling oneself. Nature 230, 598-599 (1971).

20. Crapse, T. B. \& Sommer, M. A. Corollary discharge across the animal kingdom. Nat. Rev. Neurosci. 9, 587-600 (2008).

21. Cullen, K. E. Sensory signals during active versus passive movement. Curr. Opin. Neurobiol. 14, 698-706 (2004).

22. Straka, H., Simmers, J. \& Chagnaud, B. P. A New Perspective on Predictive Motor Signaling. Curr. Biol. 28, R232-R243 (2018).

23. Schneider, D. M. \& Mooney, R. How Movement Modulates Hearing. Annu. Rev. Neurosci. 41, 553-572 (2018).

24. Brooks, J. X. \& Cullen, K. E. Predictive Sensing: The Role of Motor Signals in Sensory Processing. Biol. Psychiatry Cogn. Neurosci. Neuroimaging 4, 842-850 (2019).

25. Poulet, J. F. A. A. \& Hedwig, B. The Cellular Basis of a Corollary Discharge. Science (80-. ). 311, 518-522 (2006).

26. Poulet, J. F. A. \& Hedwig, B. Corollary Discharge Inhibition of Ascending Auditory Neurons in the Stridulating Cricket. J. Neurosci. 23, 4717-4725 (2003).

27. Schneider, D. M., Sundararajan, J. \& Mooney, R. A cortical filter that learns to suppress the acoustic consequences of movement. Nature 561, 391-395 (2018).

28. Fukutomi, M. \& Carlson, B. A. A History of Corollary Discharge: Contributions of Mormyrid Weakly Electric Fish. Front. Integr. Neurosci. 14, (2020).

29. Sawtell, N. B. Neural Mechanisms for Predicting the Sensory Consequences of Behavior: Insights from Electrosensory Systems. Annu. Rev. Physiol. 79, 381-399 (2017).

30. Cullen, K. E. The vestibular system: multimodal integration and encoding of selfmotion for motor control. Trends Neurosci. 35, 185-196 (2012).

31. Brooks, J. X., Carriot, J. \& Cullen, K. E. Learning to expect the unexpected: rapid updating in primate cerebellum during voluntary self-motion. Nat. Neurosci. 18, 13101317 (2015).

32. Roy, J. E. Dissociating Self-Generated from Passively Applied Head Motion: Neural Mechanisms in the Vestibular Nuclei. J. Neurosci. 24, 2102-2111 (2004).

33. Angel, R. W. \& Malenka, R. C. Velocity-dependent suppression of cutaneous sensitivity during movement. Exp. Neurol. (1982). doi:10.1016/0014-4886(82)90244-8

34. Chapman, C. E., Bushnell, M. C., Miron, D., Duncan, G. H. \& Lund, J. P. Sensory perception during movement in man. Exp. Brain Res. 68, 516-524 (1987).

35. Post, L. J., Zompa, I. C. \& Chapman, C. E. Perception of vibrotactile stimuli during motor activity in human subjects. Exp. Brain Res. 100, 107-120 (1994). 
36. Gertz, H., Voudouris, D. \& Fiehler, K. Reach-relevant somatosensory signals modulate tactile suppression. J. Neurophysiol. 117, 2262-2268 (2017).

37. Fraser, L. E. \& Fiehler, K. Predicted reach consequences drive time course of tactile suppression. Behav. Brain Res. 350, 54-64 (2018).

38. Voudouris, D., Broda, M. D. \& Fiehler, K. Anticipatory grasping control modulates somatosensory perception. J. Vis. 19, 1-10 (2019).

39. Williams, S. R., Shenasa, J. \& Chapman, C. E. Time course and magnitude of movement-related gating of tactile detection in humans. I. Importance of stimulus location. J. Neurophysiol. 79, 947-963 (1998).

40. Williams, S. R. \& Chapman, C. E. Time course and magnitude of movement-related gating of tactile detection in humans. II. Effects of stimulus intensity. J. Neurophysiol. (2000). doi:10.1152/jn.2000.84.2.863

41. Chapman, C. E. \& Beauchamp, E. Differential controls over tactile detection in humans by motor commands and peripheral reafference. J. Neurophysiol. 96, 16641675 (2006).

42. Cybulska-Klosowicz, A., Meftah, E. M., Raby, M., Lemieux, M. L. \& Chapman, C. E. A critical speed for gating of tactile detection during voluntary movement. Exp. Brain Res. 210, 291-301 (2011).

43. Colino, F. L. \& Binsted, G. Time Course of Tactile Gating in a Reach-to-Grasp and Lift Task. J. Mot. Behav. 48, 390-400 (2016).

44. Voudouris, D. \& Fiehler, K. Enhancement and suppression of tactile signals during reaching. J. Exp. Psychol. Hum. Percept. Perform. (2017). doi:10.1037/xhp0000373

45. Colino, F. L., Buckingham, G., Cheng, D. T., van Donkelaar, P. \& Binsted, G. Tactile gating in a reaching and grasping task. Physiol. Rep. 2, 1-11 (2014).

46. Papakostopoulos, D., Cooper, R. \& Crow, H. J. Inhibition of cortical evoked potentials and sensation by self-initiated movement in man. Nature 258, 321-324 (1975).

47. Starr, A. \& Cohen, L. G. 'Gating' of somatosensory evoked potentials begins before the onset of voluntary movement in man. Brain Res. 348, 183-186 (1985).

48. Rushton, D. N., Roghwell, J. C. \& Craggs, M. D. Gating of somatosensory evoked potentials during different kinds of movement in man. Brain 104, 465-491 (1981).

49. Giblin, D. R. SOMATOSENSORY EVOKED POTENTIALS IN HEALTHY SUBJECTS AND IN PATIENTS WITH LESIONS OF THE NERVOUS SYSTEM. Ann. N. Y. Acad. Sci. 112, 93-142 (1964).

50. Chapman, C. E. Active versus passive touch: Factors influencing the transmission of somatosensory signals to primary sornatosensory cortex. Can. J. Physiol. Pharmacol. 72, 558-570 (1994).

51. Lei, Y., Ozdemir, R. A. \& Perez, M. A. Gating of sensory input at subcortical and cortical levels during grasping in humans. J. Neurosci. (2018). doi:10.1523/JNEUROSCI.0545-18.2018

52. Azim, E. \& Seki, K. Gain control in the sensorimotor system. Curr. Opin. Physiol. 8, 177-187 (2019).

53. Ghez, C. \& Lenzi, G. L. Modulation of sensory transmission in cat lemniscal system during voluntary movement. Pflügers Arch. Eur. J. Physiol. 323, 273-278 (1971).

54. Chapin, J. K. \& Woodward, D. J. Modulation of sensory responsiveness of single somatosensory cortical cells during movement and arousal behaviors. Exp. Neurol. 72, 164-178 (1981).

55. Jiang, W., Chapman, C. E. \& Lamarre, Y. Modulation of the cutaneous responsiveness of neurones in the primary somatosensory cortex during conditioned arm movements in the monkey. Exp. Brain Res. (1991). doi:10.1007/BF00231455

56. Jiang, W., Lamarre, Y. \& Chapman, C. E. Modulation of cutaneous cortical evoked 
potentials during isometric and isotonic contractions in the monkey. Brain Res. (1990). doi:10.1016/0006-8993(90)90010-9

57. Seki, K. \& Fetz, E. E. Gating of sensory input at spinal and cortical levels during preparation and execution of voluntary movement. J. Neurosci. 32, 890-902 (2012).

58. Seki, K., Perlmutter, S. I. \& Fetz, E. E. Sensory input to primate spinal cord is presynaptically inhibited during voluntary movement. Nat. Neurosci. 6, 1309-1316 (2003).

59. McNamee, D. \& Wolpert, D. M. Internal Models in Biological Control. Annu. Rev. Control. Robot. Auton. Syst. 2, 339-364 (2019).

60. Collins, D. F., Cameron, T., Gillard, D. M. \& Prochazka, A. Muscular sense is attenuated when humans move. J. Physiol. 508, 635-643 (1998).

61. Juravle, G., Binsted, G. \& Spence, C. Tactile suppression in goal-directed movement. Psychon. Bull. Rev. (2017). doi:10.3758/s13423-016-1203-6

62. Brown, H., Adams, R. a., Parees, I., Edwards, M. \& Friston, K. Active inference, sensory attenuation and illusions. Cogn. Process. 14, 411-427 (2013).

63. Limanowski, J. et al. Action-Dependent Processing of Touch in the Human Parietal Operculum and Posterior Insula. Cereb. Cortex 30, 607-617 (2020).

64. Saradjian, A. H. Sensory modulation of movement, posture and locomotion. Neurophysiol. Clin. 45, 255-267 (2015).

65. Palmer, C. E., Davare, M. \& Kilner, J. M. Physiological and Perceptual Sensory Attenuation Have Different Underlying Neurophysiological Correlates. J. Neurosci. 36, 10803-10812 (2016).

66. Boehme, R., Hauser, S., Gerling, G. J., Heilig, M. \& Olausson, H. Distinction of selfproduced touch and social touch at cortical and spinal cord levels. Proc. Natl. Acad. Sci. 116, 2290-2299 (2019).

67. Thomas, E. R., Yon, D., Lange, F. P. de \& Press, C. Action enhances predicted touch. bioRxiv (2020). doi:10.1101/2020.03.26.007559

68. Voss, M., Ingram, J. N., Wolpert, D. M. \& Haggard, P. Mere expectation to move causes attenuation of sensory signals. PLoS One 3, 2-6 (2008).

69. Blakemore, S.-J., Smith, J., Steel, R., Johnstone, E. C. \& Frith, C. D. The perception of self-produced sensory stimuli in patients with auditory hallucinations and passivity experiences: evidence for a breakdown in self-monitoring. Psychol. Med. 30, 11311139 (2000).

70. Shergill, S. S., Samson, G., Bays, P. M., Frith, C. D. \& Wolpert, D. M. Evidence for sensory prediction deficits in schizophrenia. Am. J. Psychiatry 162, 2384-2386 (2005).

71. Shergill, S. S. et al. Functional Magnetic Resonance Imaging of Impaired Sensory Prediction in Schizophrenia. JAMA Psychiatry 71, 28 (2014).

72. Parees, I. et al. Loss of sensory attenuation in patients with functional (psychogenic) movement disorders. Brain 137, 2916-2921 (2014).

73. Wolpe, N. et al. Sensory attenuation in Parkinson's disease is related to disease severity and dopamine dose. Sci. Rep. 8, 15643 (2018).

74. Macerollo, A. et al. Dopaminergic Modulation of Sensory Attenuation in Parkinson's Disease: Is There an Underlying Modulation of Beta Power? Front. Neurol. 10, 1-14 (2019).

75. Williams, S. R. \& Chapman, C. E. Time course and magnitude of movement-related gating of tactile detection in humans. III. Effect of motor tasks. J. Neurophysiol. 88, 1968-1979 (2002).

76. Rauch, R., Angel, R. W. \& Boylls, C. C. Velocity-dependent suppression of somatosensory evoked potentials during movement. Electroencephalogr. Clin. Neurophysiol. Evoked Potentials 62, 421-425 (1985). 
77. Cohen, L. G. \& Starr, A. Localization, timing and specificity of gating of somatosensory evoked potentials during active movement in man. Brain (1987). doi:10.1093/brain/110.2.451

78. Pertovaara, A., Kemppainen, P. \& Leppänen, H. Lowered cutaneous sensitivity to nonpainful electrical stimulation during isometric exercise in humans. Exp. Brain Res. 89, 447-452 (1992).

79. Shadmehr, R., Krakauer, J. W., Neuroanatomy, A. C. \& Motor, F. O. R. A computational neuroanatomy for motor control. Exp. Brain Res. 185, 359-381 (2008).

80. Kawato, M. Internal models for motor control and trajectory planning. Curr. Opin. Neurobiol. 9, 718-727 (1999).

81. Todorov, E. Optimality principles in sensorimotor control. Nat. Neurosci. 7, 907-15 (2004).

82. Wolpert, D. M. \& Ghahramani, Z. Computational principles of movement neuroscience. Nat. Neurosci. 3, 1212-1217 (2000).

83. Bays, P. M. \& Wolpert, D. M. Computational principles of sensorimotor control that minimize uncertainty and variability. J. Physiol. 578, 387-396 (2007).

84. Bays, P. M. \& Wolpert, D. M. Predictive attenuation in the perception of touch. in Sensorimotor Foundations of Higher Cognition (eds. Haggard, E. P., Rosetti, Y. \& Kawato, M.) 22, 339-358 (Oxford University Press, 2008).

85. Wolpert, D. M. \& Flanagan, J. R. Motor prediction. Curr. Biol. 11, R729-R732 (2001).

86. Diedrichsen, J., Verstynen, T., Hon, A., Lehman, S. L. \& Ivry, R. B. Anticipatory adjustments in the unloading task: Is an efference copy necessary for learning? Exp. Brain Res. 148, 272-276 (2003).

87. Klaffehn, A. L., Baess, P., Kunde, W. \& Pfister, R. Sensory attenuation prevails when controlling for temporal predictability of self- and externally generated tones. Neuropsychologia 132, 107145 (2019).

88. Bäß, P., Jacobsen, T. \& Schröger, E. Suppression of the auditory N1 event-related potential component with unpredictable self-initiated tones: Evidence for internal forward models with dynamic stimulation. Int. J. Psychophysiol. (2008). doi:10.1016/j.ijpsycho.2008.06.005

89. Miall, R. C. \& Wolpert, D. M. Forward models for physiological motor control. Neural Networks 9, 1265-1279 (1996).

90. Wolpert, D. M., Miall, R. C. \& Kawato, M. Internal models in the cerebellum. Trends in Cognitive Sciences 2, 338-347 (1998).

91. Shadmehr, R., Smith, M. a \& Krakauer, J. W. Error correction, sensory prediction, and adaptation in motor control. Annu. Rev. Neurosci. 33, 89-108 (2010).

92. Therrien, A. S. \& Bastian, A. J. The cerebellum as a movement sensor. Neurosci. Lett. 688, 0-1 (2019).

93. Adams, R. a., Shipp, S. \& Friston, K. J. Predictions not commands: Active inference in the motor system. Brain Struct. Funct. 218, 611-643 (2013).

94. Friston, K. The free-energy principle: A unified brain theory? Nature Reviews Neuroscience 11, 127-38 (2010).

95. Pickering, M. J. \& Clark, A. Getting ahead: Forward models and their place in cognitive architecture. Trends Cogn. Sci. 18, 451-456 (2014).

96. Oldfield, R. C. The assessment and analysis of handedness: the Edinburgh inventory. Neuropsychologia 9, 97-113 (1971).

97. Core Team, R. R: A language and environment for statistical computing. $R$ Foundation for Statistical Computing (2018). doi:10.1007/978-3-540-74686-7

98. JASP \& JASP Team. JASP. [Computer software] (2019).

99. Benjamini, Y. \& Hochberg, Y. Controlling the False Discovery Rate: A Practical and 
bioRxiv preprint doi: https://doi.org/10.1101/2020.11.13.381202; this version posted May 6, 2021. The copyright holder for this preprint

(which was not certified by peer review) is the author/funder. All rights reserved. No reuse allowed without permission.

Powerful Approach to Multiple Testing. J. R. Stat. Soc. Ser. B (1995).

881 doi:10.1111/j.2517-6161.1995.tb02031.x

882 